\title{
Editorial
}

\section{Peer Review Week: Quality in Peer Review}

\section{Introduction}

At The Journal of Neuroscience, we really enjoy Peer Review Week. This week gives us the opportunity to thank our reviewers for carrying out this essential work. We continue to believe deeply that rigorous peer review improves the scientific literature. I know that my own work has benefited significantly from the input of many peer reviewers, not just in catching missed controls, but also in interpreting findings based on a different perspective or a view that is a little more distant from the data collection, or sometimes even catching a fatal flaw that sends us back to the bench. Our goal at JNeurosci is to publish papers that trainees and senior researchers can rely on, and that they feel comfortable citing in their own grants and papers because the science is held to a high standard of rigor and has lasting impact. We work hard to be the most trusted source for reading about neuroscience research, both recent and archival, so that the information published in JNeurosci remains useful many years after publication. In addition, we consider the reviewers who contribute to JNeurosci's mission to be important members of our editorial team.

This year the theme of Peer Review Week is "Quality in Peer Review." This is a timely topic for JNeurosci because our Editorial Board discusses this issue frequently and is continually working to adopt policies that will strengthen peer review quality. As you probably know, our editors are working scientists who can reflect on their own experiences with papers in the peer review process. We also have two levels of editorial oversight so that each paper has the personal attention of a Reviewing Editor who is close enough to the area of neuroscience in the paper to invite knowledgeable reviewers and evaluate the reviews and research themselves. The Reviewing Editors' decisions are then shared with a Senior Editor who can calibrate across areas of neuroscience and make sure that standards of review are applied similarly in each domain. This introduces another level of scientific evaluation. Finally, we have introduced a number of new procedures to improve peer review, including consultation sessions between discrepant reviewers to reach a consensus set of recommendations for authors, a set of standards for experimental design and statistical analyses (https://www.jneurosci.org/collection/experimental-design-editorials) so that authors and reviewers can work from the same standards. We value feedback from our reviewers, authors, and readers on how we can continue to improve the editorial process at JNeurosci.

Another important way to maintain the quality of our peer review process is to help train the next generation of fair and rigorous reviewers. We often get questions from trainees and junior colleagues asking how they can get involved in reviewing manuscripts. In response to these requests and to questions from our editors about how to increase the diversity of our reviewer pool and make sure that the standards for a constructive review are clearly laid out, we have developed a Reviewer Mentoring Program (https:// www.jneurosci.org/content/sfn-reviewer-mentor-program). The goal of the program is to provide trainees with examples of what goes into constructive and rigorous scientific review and to help develop new members of our peer review community. The program is open to SfN members who have published at least one manuscript as first author. We believe that it is essential to have gone through the peer review process and received and responded to reviews to begin to participate as a reviewer. The first step in the process is to review materials developed in collaboration with our sister journal eNeuro that describe the process and lay out the qualities of a quality review (https://neuronline.sfn.org/Articles/Professional-Development/ 2016/Tricks-of-the-Trade-How-to-Peer-Review-a-Manuscript). Next, we pair the trainee with an experienced reviewer who has consistently provided outstanding reviews for JNeurosci or eNeuro (most are current members of our editorial boards). The trainee and their review mentor look for an interesting paper posted to bioRxiv and develop a review together. Once the mentor agrees that the review is strong, the trainee graduates from the program and can post the review if they so choose. So far, we have had 41 trainees graduate from the program, and we hope that they continue to value the peer review process and become effective members of the peer review community.

Another issue I hear when there is discussion of peer review is that anonymity allows some reviewers to belittle and degrade authors. This is something we do not tolerate at JNeurosci. First, remember that reviewers are not fully anonymous: the editors know their identity, screen for inappropriate comments, and rate their reviews in our reviewer database. Second, if an editor notes rude or abusive comments in a review, we will contact a reviewer to edit it and, in an egregious case, revoke the review and blacklist the reviewer. Note that this occurs very, very rarely. The vast majority of JNeurosci reviewers are collegial and work hard to provide constructive comments. We take seriously our editorial responsibility to keep the conversation respectful.

The most wonderful thing that Peer Review Week lets us do is to thank a subset of our hard-working reviewers personally. Reviewing Editors get the chance to call out those individuals who have jumped in to fill in for a late reviewer, resolved a scientific disagreement, or simply provided consistently outstanding reviews. I can only mention a few of those reviewers each year, but I want to take this chance to thank all of you who have given your time to make sure that the papers published in JNeurosci are rigorous and will remain useful to the scientific community for many years into the future. We appreciate the many hours of work you put in to evaluate and comment on the science. Thank you!

Please send any suggestions or comments on how we can improve the peer review process at JNeurosci to JN_EiC@SfN.org or Tweet suggestions to @MarinaP63.

(D)Marina Picciotto

EiC, JNeurosci

https://doi.org/10.1523/JNEUROSCI.1987-19.2019 